\title{
Au-delà de l'intelligibilité mutuelle : l'activité collective comme transaction. Un apport du pragmatisme illustré par trois cas
}

Beyond mutual intelligibility: collective activity as a transaction. The contribution of Pragmatism illustrated by three cases

Alexandra Bidet, Manuel Boutet et Frédérique Chave

\section{OpenEdition}

Journals

Édition électronique

URL : http://journals.openedition.org/activites/632

DOI : $10.4000 /$ activites.632

ISSN : $1765-2723$

Éditeur

ARPACT - Association Recherches et Pratiques sur les ACTivités

\section{Référence électronique}

Alexandra Bidet, Manuel Boutet et Frédérique Chave, «Au-delà de l'intelligibilité mutuelle : l'activité collective comme transaction. Un apport du pragmatisme illustré par trois cas », Activités [En ligne] 10-1 | Avril 2013, mis en ligne le 15 avril 2013, consulté le 30 avril 2019. URL : http:// journals.openedition.org/activites/632; DOI : 10.4000/activites.632

\section{(c) $(7)$}

Activités est mis à disposition selon les termes de la licence Creative Commons Attribution - Pas d'Utilisation Commerciale - Pas de Modification 4.0 International. 


\title{
Au-delà de l'intelligibilité mutuelle : l'activité collective comme transaction. Un apport du pragmatisme illustré par trois cas
}

\author{
Alexandra Bidet \\ Centre Maurice Halbwachs, CNRS-EHESS-ENS, 48 bd Jourdan, 75014 Paris alexandra.bidet@ens.fr
}

Manuel Boutet

IDHE, CNRS-Université Paris Ouest-La Défense — manuel.boutet@free.fr

Frédérique Chave

Centre Maurice Halbwachs, CNRS-EHESS-ENS, 48 bd Jourdan, 75014 Paris — fr.chave@gmail.com

«Ce que nous partageons n'est pas aussi intéressant que ce que nous ne partageons pas »

(C. Bender à propos de M. Bakhtine, cité par Béguin, \& Clot, 2004)

\begin{abstract}
Beyond mutual intelligibility: collective activity as a transaction. The contribution of Pragmatism illustrated by three cases. Interactions are at the core of major sociological approaches to work. Some lines of research have accounted for cooperative interactions which include incommensurate perspectives. But in this paper we argue that the notion of interaction needs to be extended to the notion of transaction, deeply rooted in the American pragmatist tradition. The shift from interaction to transaction allows for the study of a wide range of situations lacking mutual intelligibility. The main feature is the coexistence of cooperation and entirely asymmetric perspectives, not just for a transitory moment in the process of exchanging perspectives, but as a stabilized configuration. Such contexts mean coming to terms with long unnoticed but increasingly topical components of work environments. To understand these singular forms of coordination with minimal interactions, we need to take account of the role of digital artifacts, third-party participants, and personal rhythms. This paper draws on three studies conducted in different organizational settings: a telephone traffic control center, a pediatric emergency room, and on-line gaming in the workplace. Where collective activity does not mean mutual intelligibility, the analysis turns to the various forms of life developed in the process of work, the intermittent encounters between commitments that are unaware of one another, and the workers confronted with their multiple spaces of activity
\end{abstract}

\section{KEYWORDS}

cooperation, collective activity, work, experience, transaction, pragmatism, sociology, emergency, hospital, game, control room 


\section{1.- Introduction ${ }^{1}$}

Quel est l'apport de la tradition pragmatiste à l'étude des activités collectives ? À cette tradition, on associe communément les écrits, aux États-Unis, entre la fin du $19^{\mathrm{e}}$ siècle et la moitié du $20^{\mathrm{e}}$ siècle, de C. S. Peirce, W. James, J. Dewey et G. H. Mead. Leur postérité a été pour partie documentée en sociologie générale, où l'on l'identifie à l'École de Chicago et plus spécifiquement à l'interactionnisme symbolique, mais aussi en sociologie urbaine, à travers la figure de l'étranger et l'écologie urbaine classique de Chicago, et en sociologie politique ou dans les policy sciences, quand elles s'intéressent aux mobilisations et à la construction des problèmes publics. Elle est explicite aussi au sein des communication studies (Bergman, 2007 ; Craig, 2007).

Dans le champ du travail, ou des activités ordinaires, la situation est plus confuse. D'un côté, la postérité des pragmatistes américains du début du siècle est manifeste. La seconde École de Chicago, à travers E. C. Hughes, A. Strauss, H. S. Becker et E. Goffman, a produit le premier corpus d'études ethnographiques du travail ; l'écologie des activités et les workplace studies, via E. Goffman et $\mathrm{H}$. Garfinkel, entretiennent aussi de forts liens avec la tradition pragmatiste; et l'étude des organisations a été marquée par la relecture opérée par D. A. Schön de la théorie de l'enquête de J. Dewey. D'un autre côté, l'apport des pragmatistes n'est pas identifié en propre. On évoque davantage l'interactionnisme symbolique, c'est-àdire la reformulation par $\mathrm{H}$. Blumer de développements associés à G. H. Mead. Et, quand il s'agit de faire référence à une orientation spécifique, on convoque plutôt le courant de l'«action située » (Quéré, 1997) et son « situationnisme méthodologique » (Joseph, 1998).

Dans cet article, nous suggérons au contraire l'intérêt de caractériser en propre l'apport des pragmatistes. Il en va d'un approfondissement du Practice Turn, qui tend à renouveler l'étude des organisations et d'autres objets des sciences sociales. Cet approfondissement s'impose tout particulièrement pour aborder le travail dans un monde technicisé et cosmopolite où, d'un point de vue organisationnel, une part plus grande est laissée aux improvisations du moment et aux expertises locales, et où, du point de vue des acteurs, les coopérations se tissent de plus en plus souvent avec des personnes et des dispositifs techniques qui leur sont et leur restent étrangers. Les pragmatistes, en particulier G. H. Mead et J. Dewey, offrent en effet des outils permettant de penser l'accomplissement pratique de l'activité collective, tout en sortant du seul horizon d'intersubjectivité et de réciprocité des perspectives, qui oriente la majorité des recherches 3 . Passer de la notion d'interaction à celle de transaction élargit, au-delà des configurations habituellement associées à la notion d'interaction, les situations de coopération que nous pouvons considérer. Car nous allons voir qu'il est une partie de l'action conjointe ou de l'activité collective que la notion d'interaction décrit mal et qui appelle un vocabulaire plus large.

En mettant en regard trois terrains d'enquête marqués par une forme durable de nonréciprocité des perspectives entre les protagonistes, nous illustrerons quelques apports de ce vocabulaire et préciserons les outils d'analyse que l'héritage pragmatiste invite ainsi à élaborer. Mais nous commençons par rappeler qu'à travers les notions d'interaction et de coopération, l'étude des activités collectives s'est longtemps focalisée sur la question de l'intelligibilité mutuelle.

\section{2.- De l'intelligibilité mutuelle à l'expérience : revisiter}

1 Ce travail s'est inscrit dans le cadre du projet Communication et multi-activité au travail (ANR-08-COMM039) coordonné par Alexandra Bidet.

2 « The Dewey-Mead theory translates silently into their sociology », résume A. Strauss (1992, p. 10).

3 Jusque dans l'étude de situations sans paroles ni face à face : Pagis, 2010. 


\section{l'héritage pragmatiste}

«L'intelligence de la situation n'est ni individuelle ni collective, elle ne passe pas toujours par des règles 'mutuellement admises' : l'intelligence de la situation de travail, pour ceux qui y participent, pousse ainsi, non par convergence sur un contrat, mais en réseaux plus ou moins connectés et plus ou moins denses, en 'chaînes de coopération' qui font la consistance de l'espace-temps de travail » (Joseph, 1994).

Ce que la littérature sociologique a retenu des écrits des pragmatistes du début du siècle, à travers les courants ou approches mentionnés plus haut, semble s'être classiquement ordonné autour d'une question - comment interagir, comment coopérer - et d'une façon d'y répondre, consistant à s'intéresser aux opérations qui produisent une intelligibilité mutuelle entre les participants. Dans le domaine du travail, et plus largement des activités coopératives finalisées, aucune thématique n'a suscité plus d'intérêt et de travaux : comment les participants parviennent-ils à se coordonner, à dépasser l'hétérogénéité de leurs perspectives (Bechky, 2003 ; Fligstein \& McAdam, 2012 ; Katz, 2002 ; Strauss, 1993) ?

Cette attention à l'hétérogénéité des perspectives marquait une rupture avec les conceptions cognitives et psychologiques traditionnelles, qui utilisaient les notions de représentation partagée et de but commun pour rendre compte du travail collectif (Grosjean, 2005). Suivre l'intuition pragmatiste consiste alors à étudier le travail collectif «en train de se faire » (in the making) et, pour cela, à mettre au jour les processus interactionnels et communicationnels variés qui le constituent et par lesquels s'accomplit la collaboration entre les participants. L'intelligibilité mutuelle, en particulier, se déploie progressivement. Dans ces approches centrées sur la notion d'interaction, comme avec les notions de représentations partagées ou de but commun, l'intelligibilité mutuelle reste la pierre de touche de la coopération: on se demande dans quelle mesure les perspectives des participants se rapprochent l'une de l'autre.

Décrivant une salle de contrôle aérien, Goodwin et Gookwin notent que «ce que chacun voit dans ce panoptique n'est pas une image d'ensemble, mais une diversité polymorphe de perspectives localisées » (1996). De même Joseph, en prenant appui sur la théorie des cadres d'E. Goffman, soutient que tout contexte de travail «doit être conçu comme un assemblage de cadres participatifs différents où les agents s'engagent selon des modalités diverses, devant des audiences et pour des destinataires différents » (1994). Il s'agit alors d'étudier comment les «protocoles de la coopération » sont constamment réélaborés dans le cours des activités - étant entendu qu' «il n'y a pas de pertinence par convention » et qu'elle doit donc être entretenue en situation (Joseph, \& Quéré, 2003). La gestion de l'ordre interactionnel s'apparente ainsi à un labeur indéfini, sans lequel le travail lui-même ne peut s'accomplir : il s'agit de rendre la coopération possible. Que l'on s'intéresse aux équipes de travail ou au couple agent/usager, la co-production de biens ou de services est donc toujours, dans ce cadre, celle de perspectives plus ou moins communes: une "visibilité mutuelle des situations, des gestes, des opérations dans l'espace de travail » (Quéré, 1997, p. 167), des «apparences concertées » (Joseph, 1998), un «contexte partagé » ${ }^{4}$ (Grosjean, 2005 ; Salembier, \& Zouinar, 2004), etc.

Pour ces auteurs, la notion de perspective présente le grand intérêt de déplacer l'accent de ce qui est connu vers ce qui peut être partagé. Ils suivent ainsi le projet, commun à C. S. Peirce et à L. Wittgenstein, d'éliminer la «denrée mentale » de la théorie sociale (Descombes, 1995). Il s'agit de penser la connaissance en termes de procédures plutôt que de stock, en favorisant des approches non plus déductives, mais naturalistes. Si le langage reste

4 Les deux auteurs définissent un contexte partagé comme « un ensemble d'informations ou évènements contextuels mutuellement manifestes pour un ensemble d'acteurs, à un instant $t$ dans une situation donnée, compte tenu de leurs capacités perceptuelles et cognitives, des tâches qu'ils doivent réaliser et de leur activité en cours » (Salembier, \& Zouinar, 2004, 79). Les événements contextuels sont ceux « qui se manifestent au cours d'une activité et qui sont ou peuvent être pertinents relativement à la réalisation de cette activité et ceci du point de vue des acteurs » (ibid., les auteurs soulignent). 
important, l'environnement devient toutefois le principal terrain d'investigation: on s'intéresse à ce qui est visible, et plus précisément «manifeste»- non pas seulement perceptible, mais inférable (Salembier, \& Zouinar, 2004).

«Continuellement confrontés à la tâche de juxtaposer des perspectives sur l'objet, quel qu'il soit, avec lequel ou sur lequel ils travaillent de façon à le situer dans un réseau pertinent de significations », les contrôleurs au sol étudiés par les Goodwin parviennent à accorder ou à articuler leurs vues (1996, p. 89). Il en va différemment, par exemple, dans le cas du binôme formé par les voyageurs et les agents de supervision de la ligne A du RER étudiés par I. Joseph. Ce qui fait défaut, dans ce cas, est la possibilité d'une interaction directe avec les usagers : «si l'informateur avait seulement la possibilité de voir comment des voyageurs agglutinés sur un quai réagissent à une annonce, il serait en mesure de s'adresser véritablement à un destinataire, d'observer comment il oriente ses mouvements. Il pourrait considérer l'événement (l'incident) comme 'mutuellement intelligible', évaluer la pertinence de l'action (l'initiative en matière d'information) »(Joseph, 1994, p. 583).

L'interaction sociale apparaît ainsi comme une condition souvent nécessaire, quoique non suffisante, pour que puissent se juxtaposer ou s'articuler les perspectives des participants. Ici le cadre organisationnel ne suffit pas, quoiqu'il ait sa place. L'alignement des convictions demande aussi de partager des «bribes de culture», selon une expression de J. Gumperz (1989); et ce partage peut exiger des protagonistes un mouvement d'enquête, notamment quand le «travail de publication et de mise à disposition» des intentions réciproques est défaillant, comme il peut se suffire des ressources de la co-présence, ou de la «conversation par gestes » thématisée par G. H. Mead. Quand M. Breviglieri étudie des scènes publiques d'entraide entre des usagers des automates de la SNCF, il décrit ainsi un geste «ajusté sur des formes minimales d'intercompréhension », qui implique la production d'une lecture partagée des évènements, d'une «proximité circonstancielle » entre partenaires (Breviglieri, 1997, p. 144). Il montre qu'elle requiert l'interface de l'appareil comme «terrain en commun », et qu'elle passe par «le partage de l'attention, la compréhension réciproque d'éléments contextuels, la sollicitation et la sensibilisation d'un intervenant, l'intervention elle-même, dont la nature furtive et spontanée apparaîtra décisive » (ibid., pp. 124, 127). En particulier, les relations de civilité opèrent, dans ces scènes d'entraide en public (montrer de la tolérance, inviter à l'action...), en «facilitant l'intelligibilité mutuelle d'éléments circonstanciels et soutenant la correction en public en cas d'échec opératoire » (ibid., pp. 144, 124). Si l'usage du langage est rare, la construction d'une telle proximité est plutôt affaire de rythme : la félicité du geste d'entraide, son existence même, dépend de sa capacité à se glisser dans le rythme hésitant qui succède à une déconvenue dans la manipulation de l'interface. Outre des moments d'enquêtes sur les intentions des usagers, et sur les données de la situation, afin de repérer l'origine de la déconvenue avec l'appareil, le plus décisif pour que l'entraide ait lieu relève en effet d'un tact qui est "pour l'essentiel une affaire d'ajustement de l'intervenant au rythme auquel l'opérateur conduit sa commande » (ibid., p. 143).

À défaut de cet ajustement mutuel, le geste d'entraide risque d'apparaitre comme une interférence doublement incivile, faisant peser un soupçon d'incompétence sur l'usager, et laissant supposer une action aux motivations strictement personnelles de la part de celui qui lui vient en aide. Quand M. Breviglieri examine le «tact professionnel» des agents du Samusocial, qui vont quotidiennement à la rencontre d'un sans-abri dans l'espace public (2010), il identifie aussi une forme minimale d'intelligibilité mutuelle dans leur façon de s'ajuster au rythme temporel de l'usager : d'un côté, les intervenants «prennent son pas », de l'autre, ils le dirigent insensiblement vers le camion du Samusocial, après avoir « perçu dans son absence de résistance à être accompagné une inclination à consentir à se rendre au centre d'hébergement ». Si l'intervenant «évite de l'enlacer pour le porter, mais dispense à mesure qu'il fournit son effort, des gestes discrets de soutien, comme des petites poussées pour faciliter son rétablissement », c'est que l'ajustement rythmique apparaît comme un préalable 
à une interaction qui dépasserait le corps-à-corps pour se déployer sur un plan symbolique. Dans une tradition goffmanienne matinée de comportementalisme, A. Kendon fait même, en ce sens, du rythme partagé, l'élément attestant par excellence la félicité de l'interaction : par leur «coordination rythmique» ou «synchronie interactionnelle», les «participants manifestent qu'ils partagent la même perspective sur l'interaction» (1990, p. 256). Suivant une lecture plus phénoménologique d'E. Goffman, J. Katz accorde aussi une place majeure à la dimension corporelle: son étude des émotions montre qu'un accord avec les autres et notre monde environnant se réinvente par l'intermédiaire de mouvements corporels aussi ténus que la modulation de pleurs, d'une plainte ou l'intonation d'un rire (1999).

Les exemples précédents manifestent deux grandes orientations. D'une part, une exploration renouvelée des savoirs en acte, insistant sur l'hétérogénéité des points de vue en présence. D'autre part, une focalisation sur la question de l'intelligibilité mutuelle, placée au cœur de l'étude des activités coopératives. Ces deux gestes résonnent avec la tradition pragmatiste. On sait en particulier l'importance accordée à la question de l'intelligibilité mutuelle par l'interactionnisme symbolique, qui en est pour partie issue. Mais, pour la tradition pragmatiste, la production d'une intelligibilité mutuelle, d'un rapprochement des perspectives ou d' " arrangements » articulant plusieurs lignes de travail via un effort de négociation et de persuasion (Strauss, 1993, pp. 87-93), n'est ni première ni centrale. L'enjeu est plutôt de comprendre la genèse simultanée des différentes perspectives et leur enchevêtrement. Or cette question est des plus actuelles dans un contexte où, avec l'accroissement de la division sociale du travail et la globalisation des économies, la stabilité organisationnelle fait aujourd'hui souvent figure d'exception (Beck, 2001 ; Engeström, 2008 ; Schmidt, 2012, p. 202).

Pour W. James, G. H. Mead, et A. Schütz, rappelons que l'intelligibilité mutuelle est rare, difficile à atteindre, et au mieux transitoire. Comme le relève $\mathrm{C}$. Russill, l'empirisme radical de W. James considère la façon dont nos interactions avec le monde suscitent des rencontres temporaires, dans le flux de l'expérience, entre des perspectives incommensurables: «Comment deux esprits peuvent-ils connaître une même chose ? Pour James, dire que deux pensées ou deux choses sont strictement identiques est idiot, et affirmer qu'une pensée ou une chose est identique à elle-même ne veut rien dire. La question n'apparait comme un problème philosophique sérieux que si l'on prend la distinction fonctionnelle entre sujet et objet pour une distinction ontologique qu'il s'agirait de dépasser une fois pour toutes. Or quand de telles questions surgissent, elles correspondent davantage à des problèmes pratiques de coordination de nos activités dans le monde, que d'identification d'une base rationnelle pour élaborer ces activités » (Russill, 2008, pp. 289-290, notre traduction) ! La tradition interactionniste a bien inversé la priorité, en traitant l'hétérogénéité des perspectives comme une phase transitoire en direction d'un monde commun, là où l'hypothèse pragmatiste considère au contraire l'activité collective sur une base élargie, où l'ordinaire est l'hétérogénéité des perspectives.

Dans quel cadre élargi convient-il alors de se situer? Il n'a pas été ignoré par les travaux présentés plus haut, qui ont même contribué à le développer, mais sans en tirer toujours toutes les conséquences. Ce cadre correspond à une approche dite écologique, qui ne part pas d'individus constitués, ni de milieux déjà donnés, mais du couplage structurel entre individu et milieu, par lequel ils ne cessent de se produire et de se transformer. De l'écologie urbaine de l'École de Chicago (Grafmeyer, \& Joseph, 2004; Mc Kenzie, 1924) à l'écologie des activités (Cicourel, 1987) ou de la mobilité (Joseph, 2007b, p. 12), il a fait fructifié l'héritage pragmatiste (Joseph, 1998, 2002, 2007a, 2007b ; Tracés, 2008, 2012) en alimentant des développements autour de la vigilance et de la prise (Chateauraynaud, 1997), des attachements (Breviglieri, 2004 ; Hennion, 2009), de la multi-activité (Datchary, Licoppe, 2007 ; Licoppe, 2008), des façons de s'orienter (Bidet, 2008, 2011 ; Boutet, 2006, 2008), des compétences à suivre (Berger, 2008) notamment. Il a conduit à se pencher sur les écologies informationnelles (Nardi, \&O’Day, 1999) et graphiques (Denis, \& Pontille, 2010), sur le rôle 
des détails (Piette, 2009a, b) et nourrit, par exemple aussi, la proposition d'une «Core-Task Analysis » en ergonomie (Norros, 2004). Les approches qui se sont ainsi développées posent en des termes renouvelés les questions de l'agentivité et de la temporalité : elles n'attribuent le pouvoir d'agir en propre ni à l'individu ni au milieu; et rapportent tout moment à une situation en développement (Bidet, 2012; Simpson, 2009). Or c'est précisément en ce sens que J. Dewey proposait de substituer au concept d'interaction celui de transaction.

Là où « la phase subjectiviste de la philosophie européenne » (Dewey, 1993, p. 92) a promu un individu isolé et désengagé - et comme détaché de structures organisationnelles et étatiques alors pensées comme d'un autre niveau, la notion de transaction affirme au contraire la primauté du couplage de l'organisme et de l'environnement, véritable «partenaire » (Quéré, 2006). Soutenir que «l'organisme n'existe en tant que tel que dans les connexions actives avec son environnement » (ibid.), c'est alors rappeler que l'on ne vit pas seulement dans un environnement, mais par lui (Joas, 1999). L'attention aux «états de choses » (《states of affairs») de l'environnement n'attribue pas une agentivité à une entité distincte et constituée: "l'agentivité ne désigne pas un attribut, mais le mouvement des reconfigurations du monde » (Barad, 2003, p. 818, notre traduction) ; « elle ne réside pas en nous ni dans nos artefacts, mais dans nos intra-actions » affirme aussi L. Suchman (2007, p. 285, notre traduction). L'attention à cette dynamique permet donc de montrer que les acteurs ne sont pas des atomes individuels. L'environnement apparaît bien comme une entité indissociable de notre intentionnalité incarnée (Dreyfus, 1991). Si l'écologie de la perception associée au nom de J. J. Gibson est «implicitement pragmatiste », comme l'écrit I. Joseph, c'est que l'affordance est de même «une propriété qui ne saurait être attribuée ni à l'environnement ni à l'agent, mais à la relation qu'ils entretiennent » (cité in Breviglieri, \& Stavo-Debauge, 2007). Ainsi une approche écologique «traite l'organisme-dans-sonenvironnement comme une totalité indivisible, non comme la composition de facteurs externes et internes» (Ingold, 2001, notre traduction). Elle sape les interrogations aporétiques (encore chroniques : par exemple in Kaptelinin, \& Nardi, 2006, 226) sur la part du sujet et de l'environnement dans le contrôle de l'action, au profit de la dimension créative de l'agir longtemps marginalisée par les modèles de l'agir à visée normative et de l'agir instrumental (Joas, 1999 ; Emirbayer, 1997).

La conséquence directe en est aussi de penser l'organisation sur un mode plus immanent. La notion de transaction invite ainsi à analyser l'activité collective plus largement, à partir de l'expérience - et non pas seulement d'une intelligibilité mutuelle qui serait à construire. Pour J. Dewey, l'expérience correspond à l'établissement d'une «relation sentie entre faire et subir lorsque l'organisme et l'environnement interagissent» (2005, p. 253). Les significations ne se réduisent pas alors à des symboles partagés : au sein de la «contribution cumulative et réciproque entre ce qui est fait et ce qui est éprouvé », écrit J. Dewey, «c'est à l'étendue et au contenu de ces relations que l'on mesure le contenu signifiant d'une expérience » (ibid., p. 76, 69). La construction d'une perspective commune, qui a concentré l'attention de bien des travaux, apparaît alors comme un cas particulier du phénomène plus général de créativité de l'agir, qui se manifeste par la production d'habitudes, d'intérêts et de façons de s'orienter, lesquels sont bien sûr susceptibles d'être transposés d'un contexte à un autre.

Passer de l'interaction à la transaction en rattachant la question de la coopération à celle de l'expérience permet de documenter des phénomènes qui le sont encore peu. Nous allons l'illustrer à partir de trois enquêtes de terrain menées respectivement dans la téléphonie, au sein de services d'urgence hospitaliers et sur la pratique de jeux en ligne au travail. Ces trois cas ont en commun de nous confronter à des activités collectives et organisées, mais qui se sont stabilisées en dehors d'un horizon d'intelligibilité mutuelle, sans le travail d'articulation bien décrit par A. Strauss (1993). Étudier les transactions à l'œuvre donne par contre toute leur place à des aspects encore peu étudiés, mais au rôle croissant dans les contextes contemporains : l'autonomie des dispositifs numériques, tels de véritables objets-en-action, l'activité de tiers et enfin l'élaboration de rythmes personnels. Nous abordons ces trois 
dimensions successivement, en mettant l'accent sur les formes originales d'activité collective dont elles participent.

\section{3.- De la prise en compte des objets-en-action aux formes de vie développées au travail}

«L'écologie des activités analyse d'abord des échanges explicites ou furtifs, verbaux ou posturaux, entre personnes présentes dans un champ de visibilité. Elle doit aujourd'hui tenir compte des formes d'accessibilité liées aux technologies de la téléaction et du télétravail qui tentent de reconstituer les conditions du face-à-face et de la conversation ordinaire. Elle tient compte également de l'environnement physique et sensible grâce auquel se déroulent ces échanges. Enfin, elle prend en compte les objets auxquels les participants font jouer un rôle, les sollicitant pour agir, contrôler ou anticiper leur action » (Joseph, 1998).

Sur le premier terrain considéré, un centre de supervision du trafic téléphonique, la notion de transaction opère un déplacement du regard par rapport aux approches classiques en termes d'expertise technique - il n'y a pas qu'une seule (bonne) manière de comprendre et de manipuler une technique. Ce déplacement permet de comprendre l'activité en intégrant à l'analyse le rôle des automates ainsi que les différentes façons dont les travailleurs y font face. En effet, la division du travail à l'œuvre au sein du centre de supervision étudié entre deux groupes d'agents occupant formellement le même poste ne consiste pas simplement à additionner et à articuler des contributions différenciées et complémentaires. C'est qu'un tel contexte de travail, où l'agir est à ce point distribué, ne permet plus d'ignorer les transactions avec le milieu : ces agents ne sont ni dans la situation d'utiliser des outils, qu'ils pourraient manipuler à volonté, ni aux prises avec d'autres intervenants, avec qui se coordonner et élaborer un «contexte partagé »; ils sont confrontés à la nécessité de s'associer, de s'intégrer à un ensemble d'entités interdépendantes, qui dessinent un véritable monde-écran ${ }^{5}$ de signaux, de mesures, d'alarmes et de commandes informatiques.

En effet, là où le travail repose d'abord sur la dynamique systémique et cumulative d'automatismes, interagissant continument les uns avec les autres et s'auto-régulant largement, le travail humain devient activité de supervision ou de régulation. Il en est ainsi aujourd'hui d'un nombre croissant de secteurs industriels et tertiaires - tels que le pilotage aérien, la conduite de processus dans le nucléaire, la chimie -, où les infrastructures productives sont de plus en plus automatisées et numériques. Suppléer momentanément les automatismes, contrarier leur dérive structurelle, c'est alors co-agir avec eux. La continuité du processus repose in fine - ou en dernier recours - sur l'intervention active de l'opérateur humain dans ce co-fonctionnement. Observant une situation de ce type dans la téléphonie, nous avons montré que le principal enjeu n'était pas pour les travailleurs de produire entre eux un «contexte partagé », mais d'entretenir une sociabilité avec les automatismes. Ces derniers ne sont pas des objets-dans-l'action, c'est-à-dire des « outils» et des objets d'usage destinés à équiper l'action humaine. Ils sont des objets-en-action doués d'une autonomie de mouvement et dont le fonctionnement n'est pas préétabli. G. Simondon souligne que la part d'indétermination d'un ensemble technique tient à son ouverture et à sa sensibilité à son environnement (1989) ${ }^{6}$. Or aux yeux des techniciens qui en ont la charge, les dispositifs qui

5 La notion de monde-écran désigne, au-delà de l'interface informatique, « un environnement complexe composé de régions visitables et d'horizons qui fondent les activités », donc un espace dynamique, persistant, qui peut être parcouru, exploré, où de nouvelles fenêtres peuvent être ouvertes. Il concentre, en même temps que «les activités, temporalités et situations autrefois éparses des agents », toutes les possibilités de manipulation et les informations disponibles en temps réel. Voir : Knorr-Cetina, \& Brueggers, 2003, pp. 122129.

6 «Une machine purement automatique, complètement fermée sur elle-même, dans un fonctionnement prédéterminé, ne pourrait donner que des résultats sommaires. La machine qui est douée d'une haute technicité est une machine ouverte », écrit en ce sens G. Simondon. 
produisent et régulent le trafic téléphonique sont de tels ensembles complexes, qui génèrent des questions, «ce sont des processus et des projections plutôt que des choses définitives » (Knorr-Cetina, 1998). Face à ces véritables objets épistémiques, les solutions doivent être construites et ne sont pas nécessairement uniques.

Même en l'absence d'incidents, les opérateurs déploient vigilance et effort d'exploration. Pour comprendre ce souci dont ils témoignent, il faut laisser la question de la coopération pour celle de l'expérience. Ce n'est pas en effet en élaborant des appuis qu'ils parviennent à agir, ponctuellement, avec cet agrégat systémique et constamment changeant qu'est le trafic téléphonique, mais en développant une forme de vie caractérisée par ce que K. Knorr-Cetina appelle une « sociabilité avec les objets » [sociality with objects] (1997). Ce qui s'observe ici est une exploration continue du monde-écran. Ainsi, l'activité de ces agents conjure le risque du désœuvrement et de l'ennui par la recherche et l'entretien d'une occupation intéressante : « trouver ce qu'il y a d'intéressant là-dedans », « ce qu'il se passe d'intéressant », suppose une exploration continue. Seule une circulation permanente dans le réseau, en multipliant les points de vue sur «ces trucs qui marchent tout seuls », en amorçant des intrigues typiques, permet en effet, lorsqu'un incident survient, d'être en situation d'accorder son mouvement avec celui des automatismes. Comme le note J. Dewey, « ce qui est donné ici et maintenant s'enrichit de significations et de valeurs tirées de ce qui est en fait absent et seulement présent par l'imagination - en particulier tiré des expériences antérieures, c'est-à-dire du résultat consolidé des interactions antérieures avec l'environnement » $(2005$, p. 317). Il en va ici de la possibilité même d'une expérience : pouvoir développer, selon l'expression de J. Dewey, des « lignes d'intérêt actives ». Knorr-Cetina et Bruegger pointent aussi en ce sens la nécessité d'élargir l'écologie des activités aux «réalités électroniques, puisqu'elles deviennent un habitat pour certains d'entre nous » (2003, p. 126).

À l'ère numérique, cette distribution «forte» de l'agir est au cœur des écologies informationnelles complexes en plein essor (Nardi, \& O’Day, 1999). Saisir les formes de vie qui accompagnent cette multiplication de «post-social» ou «object-centered relations » (Knorr-Cetina, 1997) implique de porter autant d'intérêt sociologique à nos façons d'entrer en relation avec notre environnement qu'à la coordination entre les personnes. Au sein même du Pratice Turn, le sentiment d'une lacune relative à l'usage des techniques, à la technique comme expérience, a fait son chemin. Lucy Suchman propose ainsi de substituer au terme d'interface «homme-machine» («human-machine interchange») celui de transaction, afin de repenser les circuits par lesquels nous entrons en relation avec nos machines (Suchman, 2007, p. 285). Paul Dourish identifie quant à lui l'interaction avec «les choses elles-mêmes»comme le premier objet pour qui veut étudier l'«Embodied Interaction », définie comme "la création, la manipulation et le partage de sens à travers l'engagement dans une interaction avec des artefacts » (Dourish, 2001, notre traduction). À mesure que se multiplient les « objets techniques » plus autonomes dans leur fonctionnement et articulés entre eux, il nous faut ainsi explorer des liens nouveaux, et des horizons d'activité «pour partie libérés des contraintes de l'espace ordinaire»: «le réseau [technique] tend à se substituer au territoire comme base topographique de la solidarité de société » (Dodier, 1995, pp. 15, 350). Si, plus généralement, l'intellectualisation des situations de travail voit nos relations avec les «choses physiques» — «sociality with objects » - prendre une nouvelle ampleur, on saisit toute l'actualité du pas de côté auquel invite le concept de « transaction » proposé par J. Dewey.

Comment l'unité d'une expérience, un engagement cohérent et cumulatif sont-ils en effet possibles dans un tel milieu de travail ? Le monde-écran du trafic téléphonique, comme celui du marché financier étudié par Knorr-Cetina et Bruegger (2003), est un environnement particulièrement dispersif qui «apparaît continuellement par bribes »: il ressemble «à un tapis dont des petites sections sont déroulées devant nous (...) nous pouvons marcher dessus, nous pouvons changer de position dessus. En même temps, nous devons imaginer le tapis se constituant à mesure qu'il est déployé » (pp. 126-127). Dans notre cas, l'identification même 
du trafic téléphonique comme objet de travail suppose l'effort quotidien des agents pour articuler leurs circulations médiatisées comme une seule et même transaction avec cet agrégat synthétique et probabiliste des appels téléphoniques. Il leur faut travailler à relier continument, pour eux, les entités hétérogènes du monde-écran. Tous ne s'astreignent toutefois pas à ce travail : certains déploient leur activité en dehors du monde-écran. Ils s'attachent à acquitter régulièrement les alarmes, dans une logique de vérification ponctuelle, là où leurs collègues explorateurs suivent les courbes du trafic, dans une logique d'anticipation continue. Dans les deux cas, chacun peut ainsi espérer s'engager dans un espace dispersif sans pour autant fragmenter son activité : l'engagement de chacun a la continuité, ou plus exactement la rythmicité - «alternance de concentrations et de relâchements » (Dewey, 2005) propice à l'unité d'une expérience. Les transactions suivent des rythmicités opposées de part et d'autre, les uns offrant leur vigilance à la redondance des alarmes ordinaires et à leur traitement routinier, les autres consacrant un effort continu à être prêts à répondre à une éventuelle « crise de trafic », avec ses réactions en chaîne.

C'est parce que les premiers privilégient les messages répétitifs qui encombrent la fenêtre d'alarmes, que les seconds peuvent s'immerger dans la circulation du trafic : «pousser plus loin la vue du réseau », car «quand tu es parti sur un problème, le temps passe vite ». Réciproquement, c'est parce que les seconds s'immergent dans le monde-écran qu'ils peuvent libérer les premiers de la gestion des situations de crise et de la nécessité d'entretenir une intimité avec des automates capricieux, dans une expérimentation indéfinie. Ce faisant, les uns et les autres ménagent aussi plus largement la continuité de leurs transactions avec un milieu technique - dans le temps (entre leurs activités passées et présentes) et dans l'espace (entre travail et hors travail): les premiers s'emploient globalement à habiter des technologies électroniques, dites aussi «de la représentation », et les seconds, des technologies mécaniques, dites «de l'intervention». De ces formes de vie (ou styles) opposées - qui ne sont pas réductibles à des clivages générationnels - témoignent les vocabulaires des uns et des autres (Bidet, 2010), leurs mises en récit (Bidet, 2007), leurs représentations graphiques de l'activité (Bidet, 2008), mais aussi leurs conceptions de la «vraie technique» ou du «vrai boulot» (Bidet, 2011a). Ces valorisations contrastées, touchant au détail fin de l'activité, sont le produit de la cumulativité des transactions. Ces valorisations renvoient à la sélection et au développement, parmi les gestes possibles, d'une certaine manière d'agir et d'appréhender la technique, qui tient autant du sujet que de l'objet, autant des personnes que des artefacts. Si les vocabulaires engagent chaque fois un rapport personnel à la technique, il doit en somme peu à la psychologie individuelle, et beaucoup à la genèse normative par laquelle ces techniciens habitent leur milieu de travail. Le temps contribue ainsi doublement à maintenir le clivage entre les deux postures : par des histoires professionnelles bien différentes et, dans la salle de supervision, par les temporalités distinctes sur lesquelles les activités sont menées.

Entre ces deux formes de vie, ne s'observent ni une relation de coopération (même tacite, ce qui amènerait à parler de travail en équipe) ni une forme de concurrence ou de rivalité (ce qui supposerait une orientation mutuelle forte, à défaut de la production d'une intelligibilité mutuelle) : ni la propension à «se mettre à la place de l'autre », inhérente à la coopération, ni la tendance à déprécier l'activité de l'autre, inhérente aux situations de rivalité. Si, comme le rappelle D. Cefaï, la réciprocité des perspectives est « une affaire d'interconnexion entre motifs et objectifs, d'intercompréhension entre réflexions et projets » (1998, p. 107), c'est bien plutôt ici une non-réciprocité des perspectives qui accompagne la distribution de l'agir. La délégation tacite de gestes et de préoccupations que nous observée est émergente : elle n'est pas le fait d'une coordination, ni n'est même vraiment aperçue comme telle; la «mutual awareness » est minimale, voire inexistante. Contre le risque de dispersion, de fragmentation de l'expérience, ce qui permet de garder le fil ne renvoie ainsi à aucun des deux grands modèles, qui privilégient soit l'opérateur soit la distribution de son activité sur son environnement et/ou une équipe de travail. Dans la configuration étudiée, l'asymétrie 
des perspectives amène par contre à introduire la notion de tiers, car les deux ensembles d'agents repérés opèrent comme des tiers les uns vis-à-vis des autres.

L'attention aux transactions n'invite ainsi pas seulement à prendre davantage en compte les objets-en-action, doués d'une autonomie, et les formes de vie qui s'élaborent avec eux, mais aussi la présence de tiers en situation. C'est à partir d'un autre terrain d'enquête que nous allons envisager plus précisément leur rôle dans de telles configurations.

\title{
4.- De la prise en compte des tiers à la coïncidence momentanée des engagements
}

\begin{abstract}
«Pour un grand nombre de situations professionnelles et organisationnelles dans lesquelles les acteurs sont engagés, ce sont l'action et l'activité des tiers qui fournissent l'orientation principale dans la production pratique de la conduite, et ce sont les contributions de ces tiers qui définissent les contingences que les acteurs doivent gérer » (Heath, Hindmarsh, \& Luff, 2000, p. 672, traduit in Salembier, \& Zouinar, 2004, p. 69).
\end{abstract}

Sur le deuxième terrain envisagé, celui des urgences hospitalières pédiatriques, la notion de transaction opère aussi un décentrement par rapport aux approches classiques en termes d'interactions. Il s'agit en effet de situations où l'activité repose de façon constitutive sur plusieurs types de participants, mais en dehors d'un horizon de réciprocité des perspectives. Ce déplacement permet d'intégrer à l'analyse le rôle d'acteurs rarement considérés et les activités organisées auxquels ils prennent part. Les analyses de la coopération à l'hôpital (Goffman, 1979 ; Grosjean, \& Lacoste, 1999 ; Peneff, 2000 ; Strauss, 1992) tendent en effet à privilégier les moments où médecin et patient sont ensemble et tentent de se comprendre, à en étudier les stratégies réciproques, les ratés et les ajustements. Si ces interactions sont importantes, les chaînes de coopération sur lesquelles repose la prise en charge dépassent toutefois les seuls moments de coprésence entre patient et professionnel. D'abord, la plupart des situations n'impliquent pas seulement un professionnel et un patient, mais aussi des «tiers », les parents (Chave, 2010). Leur intervention discrète et soutenue tout au long de la prise en charge y relève d'un double «care », soignant et attentionné, allant parfois jusqu'à des soins infirmiers plus techniques et des gestes médicaux. Cette part que prennent les parents à l'activité collective est à la fois peu remarquée et indispensable. Autour de l'enfant-patient se déploient ainsi les deux engagements hétérogènes et parallèles des parents et des professionnels.

Certes, une partie de l'activité des parents est contrôlée et coordonnée par les professionnels, avec tout l'éventail des manières de fixer des coopérations, de les reconnaître et de les inscrire dans le cadre régulateur et prescriptif du service d'urgence. Mais une grande part de leur activité échappe largement à l'attention des professionnels. Elle ne se révèle qu'en creux, en cas d'absence (ou de défaillance) du parent : lorsque ce qui se faisait à bas bruit ne se fait plus et devient pour les soignants un « supplément de travail » pouvant désorganiser le service?. Cette «co-activité » est structurelle : les activités de chacun sont nécessaires à la prise en charge. Mais ces situations, comme formes d'interaction stabilisées, et non transitoires, restent en deçà d'un travail d'équipe, lequel supposerait un ajustement moment par moment des procédures communes, voire l'interchangeabilité des activités et leur prise en charge à plusieurs. On observe au contraire peu d'interactions en face à face et une importante asymétrie des perspectives. La notion de transaction permet ici de ne pas s'en tenir aux seules interactions. Même s'il arrive souvent qu'il y ait des recadrages au cours de l'épisode passé aux urgences, que des quiproquos donnent lieu à des ajustements, etc., on veut souligner l'existence de formes hybrides et spontanées de coopération dans lesquelles les interactions entre public et professionnels ne sont qu'intermittentes et se passent souvent

7 Réciproquement, le parent, en venant aux urgences, passe provisoirement le relais de sa prise en charge totale de l'enfant au médecin, ce qui relève aussi d'une forme de coordination. 
d'efforts pour rapprocher les perspectives des participants. Entre le public et les médecins, s'observent même des rythmes opposés.

Dans les deux services d'urgences pédiatriques étudiés, les patients sont examinés une première fois par un infirmier, les uns après les autres, puis un médecin examine le patient, s'il y a lieu de faire des examens, il les prescrit au personnel soignant et prend un autre dossier en attendant le retour des examens du premier patient, et ainsi de suite. Il peut ainsi se trouver avec trois, quatre, voire cinq dossiers en même temps - auxquels correspondent autant de patients à différents moments de leur prise en charge. Cette multi-activité conduit les médecins à morceler en différentes séquences le suivi de chaque patient. Ils ne suivent pas les patients de façon linéaire, de leur arrivée à leur sortie, mais séquentiellement, circulant d'un box à l'autre, en ignorant le plus souvent tout de l'activité des parents. Pour le public, en revanche, les urgences sont ainsi un lieu où l'on attend, dans la salle d'attente puis dans un box. Cette attente n'est pas seulement le fait d'une faible absorption du flux des arrivées. Elle est également constitutive du soin : périodes d'observation, temps de passage d'une perfusion, retour d'examens, sont des moments incompressibles. Aux temps propres des examens s'ajoute le temps que le médecin consacre à ses autres patients et aux cas plus urgents qu'il prend en charge à tout moment au pied levé. En cas d'affluence, tandis que le rythme de l'équipe se contracte au maximum, celui du public se dilate à l'extrême. L'augmentation du nombre de patients ne change pas foncièrement le travail des professionnels (enchaîner les actes et les consultations), mais altère son cadre d'exercice (le temps qu'il peut consacrer à chacun, la précision et la rapidité de son évaluation du degré d'urgence). Cet écart des rythmes peut se traduire par des temps d'attente de plusieurs heures, en salle d'attente puis dans les boxes et constitue une source de tensions constante avec un public dont l'attente augmente l'inquiétude, l'inconfort et la douleur. La multiactivité médicale, centrée sur l'évaluation rapide et le traitement multiséquencé d'une multiplicité de patients, suivant un tempo rapide et morcelé, contraste avec la participation souterraine et continue des parents, suivant un rythme très lent et centré sur le maintien des conditions d'existence de l'enfant. Cet écart des rythmes et des activités signale ainsi un écart de perspectives : le parent ne voit pas ce que fait le médecin, ni pourquoi on le fait attendre ; le professionnel ne perçoit pas ce qui se passe dans les boxes, ni le détail des soins que le parent peut prodiguer ou non à l'enfant-patient.

Or c'est cette asymétrie des perspectives entre tiers et professionnels qui permet une prise en charge globale de l'enfant. D'abord, les parents assurent un care à la fois domestique et soignant, qui relève du «nursing », de la surveillance aux repas, du soin «attentionné », qui prépare l'installation dans le service pour plusieurs heures, mais aussi la sortie, et réalisent un ensemble de soins infirmiers allant de la prise d'aérosols à l'administration de médicaments, en passant par la surveillance de l'évolution de l'état de l'enfant. Ces interventions du parent, sauf défaillances de sa part, ne sont ni mutualisées ni signalées ; tout en concourant à la prise en charge du patient, elles suivent un cours parallèle aux interventions des professionnels. Plutôt qu'un souci d'intelligibilité mutuelle ou de réciprocité des perspectives, on observe un hiatus entre les états émotionnels des proches, des patients et des professionnels, et leurs appréciations de ce qui est urgent ou légitime.

Les parents, en prenant soin de l'enfant, organisent sa continuité biographique. Leur perspective étant celle du temps long, ils peuvent synthétiser auprès des professionnels toutes sortes d'informations chronologiques et détaillées, souvent déterminantes pour établir un diagnostic ou un traitement. Ils opèrent également comme des alarmes, des reminders (Datchary \& Licoppe 2007) parfois insistants et plus ou moins bien accueillis. En particulier, si l'état de l'enfant s'aggrave ils peuvent sortir du box et tenter d'alerter quelqu'un. Et les médecins s'appuient sur cette possibilité pour ignorer ce qui se passe dans les box en leur absence et se consacrer aux autres patients. De même, ce sont eux qui alimentent le dossierpatient et mettent les informations en perspective, les trient, les ordonnent, les rappellent au médecin, à qui le fait de traiter chaque jour un grand nombre de personnes ne permet pas 
d'identifier individuellement chaque patient ni de se souvenir de chaque cas. Ces tiers, par leur présence continue, vigilante, permettent au médecin la pratique d'un morcellement, comme technique efficace de traitement d'un grand nombre de patients. Par delà la présence en pointillé des professionnels, l'entourage assure ainsi la continuité du care, mais aussi celle du cas pour les professionnels.

Cet entrelacement des rythmes et des tâches se passe en général d'une explicitation des droits de l'autre partie à agir sur le patient. Et bien souvent, nul besoin de s'entendre sur les valeurs, les orientations, les croyances, pour réaliser un «accord pratique » minimal sur ce qu'il faut faire. L'aspiration commune à soigner l'enfant structure les activités croisées des parents et des médecins. C'est au bien de l'enfant qu'en appellent les uns et les autres en cas de désaccord sur l'évolution et la tournure de la prise en charge. Et si l'espace aussi est cadrant, avec ses affichettes, ses couloirs dont le sol est signalisé de flèches et de sens interdits, ses équipements incitant à s'asseoir, attendre, s'approcher, toute une partie de ce qui est fait entre patient et entourage ne se laisse pas réduire à ce qui est inscrit, proscrit ou prescrit ni à ce que l'environnement suggère de faire ou de ne pas faire. Se lit à cet égard une certaine forme d'indifférence mutuelle des médecins et des parents quant à la manière dont chacun «tient son rôle», dans les longs moments où ils n'interagissent pas directement. Ce qui se passe dans le box entre les visites des professionnels est soigneusement ignoré, aux deux sens du terme. Cette indifférence établit de facto une forme de répartition des préoccupations qui laisse dans l'ombre le contenu même de ce qui serait de la responsabilité de chacun. Par cette économie de l'attention, le médecin, qui doit traiter de nombreux patients, n'a pas pour autant à se préoccuper en permanence de chacun d'eux : d'autres s'en chargent. Il s'agit d'une indifférence non pas morale, mais pratique, qui ne témoigne pas d'un désintérêt, mais bien plutôt d'une organisation de l'activité qui intègre implicitement une économie distribuée de l'attention impliquant des tiers. Notre approche révèle ainsi une activité collective caractérisée par une ténuité interactionnelle, l'hétérogénéité des acteurs, mais surtout, et, c'est ce qui rend la forme originale, l'absence d'intégration a priori dans un système procédural commun de toute une part de la participation des tiers.

Une telle forme de coordination, fondée sur une coüncidence des engagements, peut être rendue visible au moyen du concept de transaction. Celui-ci permet en effet de s'intéresser aussi à cette part des échanges pour partie indépendante de leur épaisseur relationnelle. La prise en charge comme activité se joue ainsi en dehors des moments d'ajustement entre chacun: dans le rapport différencié de chacun au patient, à son environnement et à ses propres fins. Elle se réalise sur fond d'ignorance et de confiance mêlées quant à ce que font les autres, mais aussi grâce à ce que font les autres. En cela c'est bien une coopération. Les services d'urgence pédiatrique fonctionnent en comptant sur le fait que les parents s'occuperont de toute une partie des tâches qu'un enfant exige, et les parents comptent sur les médecins et les soignants pour comprendre et résoudre la crise qui les amène, mais ni les uns ni les autres ne cherchent à s'informer mutuellement de ce qu'ils sont en train de faire. C'est donc une forme de coopération où «l'indifférence pratique » est une condition de la poursuite de l'activité. Dans ces situations d'interactions successives, simultanées, courtes, non réitérées et nombreuses, le défaut de réciprocité des perspectives s'avère une condition de l'unité de l'expérience, aussi bien pour les professionnels que pour les tiers ${ }^{8}$.

Des interactions aux transactions : cet élargissement semble particulièrement ajusté aux transformations structurelles que connaissent les organisations contemporaines. Cet apport de la tradition pragmatiste à l'étude de l'activité collective rend en effet plus attentifs à l'agentivité des objets-en-action et à celle des tiers. Dans les enquêtes de terrain présentées ci-dessus, la place faite aux phénomènes de rythmicité suggère qu'il offre aussi des outils

8 A contrario, M. Grosjean souligne d'une façon très intéressante le lien entre le privilège donné à l'intelligibilité mutuelle par les approches ethnométhodologiques et leur sous-qualification de l'activité (2005, p. 85). 
pour une meilleure prise en compte de la temporalité, associée à la notion d'expérience (Bidet, 2011b ; Quéré, Terzi, 2011 ; Rosa, 2010). Notre dernier cas va l'illustrer.

\section{5.- De la prise en compte des rythmes personnels à l'entrelacement des activités}

«Toutes ces interactions qui amènent l'ordre et la stabilité dans le flux tourbillonnant du changement sont en fait des rythmes » (Dewey, 2005, p. 35)

Dans les deux cas présentés, la supervision téléphonique et les urgences hospitalières, nous avons déjà constaté des rythmes opposés et des déphasages temporels entre les participants, et montré le rôle de tiers pour la cohérence de l'expérience de travail. La gestion de la multiactivité à l'échelle d'un service passe alors par l'entretien d'une asymétrie des perspectives entre deux catégories de participants 9 . Le troisième terrain que considèrerons, un jeu sur internet pratiqué depuis le lieu de travail, va permettre d'approfondir à l'échelle du travailleur cette analyse des phénomènes rythmiques et temporels. Ce jeu, présenté ailleurs (Boutet, 2008), a une structure commune à de nombreux jeux web. Il relève de ce que nous appelons les «jeux de rendez-vous » (Boutet, 2011), qui ne réclament pas une interactivité constante avec une interface de jeu, mais des moments de connexion. Il s'agit, dans le cas présent, de dépenser chaque jour ses « points d'action » pour chasser des monstres dans des souterrains en se connectant quelques minutes par jour. La présence de l'autre est un attribut de ces jeux, contrairement à d'autres jeux sur ordinateur auxquels l'on joue seul : choisir de jouer en ligne, c'est ici choisir de jouer avec d'autres et être prêt à coopérer avec quiconque sera disponible. Et, pour ceux qui jouent sur leur lieu de travail, le caractère régulier et quotidien du jeu est essentiel. Dans leurs relations avec leurs partenaires, l'accent est mis sur la commune disponibilité que requiert cette activité en ligne, et le haut degré d'intégration et de dépendance réciproque qu'elle demande. Les joueurs doivent partager des rythmes compatibles, suivant une configuration qui diffère du modèle des «sociabilités affinitaires » : les partenaires sont à la fois des équipiers sur lesquels compter et des gens que l'on connaît peu, avec lesquels on n'échange guère à propos de son travail ou de sa vie personnelle.

La notion de transaction aide ici à opérer un déplacement par rapport aux approches classiques des environnements de travail, en permettant de considérer, en deçà de la distinction entre «travail» et «hors travail », l'enchevêtrement des activités menées en présence et à distance. L'entrée du jeu sur internet met au premier plan les tensions liées à la multiplicité des activités, et conduit à observer que la création de formats, de routines ou de dispositifs pour traiter ces tensions n'épuise pas nos manières de mettre en regard nos activités, de les évaluer et de tenter ainsi de les hiérarchiser (Lahlou, 2000 ; Licoppe, 2008, 2009). La notion de transaction permet en effet d'explorer plus avant les façons dont nous faisons face à des écologies (de travail, mais pas seulement) de plus en plus riches en sollicitations hétérogènes et concurrentes, donc en «travail de soi » (Bidet, 2011a;Hughes, 1996).

Comment jeu et travail coexistent-ils ? D'abord, on constate que le jeu est pratiqué différemment au travail et hors travail : au travail, son rythme se cale sur celui du travail. Les deux cours d'action coexistent en parallèle sans être simultanés ni séquentialisés : dans le premier cas, le travailleur devrait jongler ou se contorsionner; dans le second cas, le jeu serait une simple affaire de «pauses ». Or les activités de jeu et de travail apparaissent plutôt désynchronisées, et d'une façon différente selon le caractère plus ou moins hétérogène $\mathrm{du}$

9 Dans les situations de multi-activité proprement dites, une « grappe d'activités reste pertinente dans son ensemble » (Datchary, \& Licoppe, 2007, p. 21). Des préoccupations d'arrière-plan demeurent donc pertinentes à côté de l'activité focale et sont susceptibles d'être ravivées à tout instant (Licoppe, 2008). 
contenu du travail.

Dans les contextes où le travail est très hétérogène, s'observe un resserrement de l'activité autour d'un unique moyen de communication, qui créé une continuité entre les tâches du jeu et celles du travail : toutes les sollicitations arrivent de la même manière et sont gérées avec les mêmes outils. Par exemple, les joueurs qui reçoivent des demandes de clients, essentiellement par courriel, se font volontiers «notifier» la présence distante du monde du jeu, et le jeu devient ainsi «un mail parmi d'autres». Dans un autre cas, un enquêté est averti à l'écran lorsqu'il reçoit un message instantané, qu'il vienne de l'un des canaux de communication qu'il entretient avec des groupes d'experts dans le cadre de ses missions professionnelles, ou du canal parallèle ouvert sur le même logiciel pour échanger avec ses partenaires de jeu. Le jeu ne désigne donc pas un nouvel ordre de sollicitations, concurrent, mais une sollicitation parmi d'autres. Dans le cas d'un travail au contenu homogène, on note plutôt une tendance à créer un cloisonnement entre jeu et travail en répliquant, pour le jeu, le dispositif existant pour le travail - par exemple une seconde boîte mail. De même, les joueurs préfèrent aller sur internet pour jouer plutôt que de recourir à des notifications les informant par mail d'un évènement survenu dans le jeu, ou à une messagerie instantanée où leurs partenaires de jeu pourraient les solliciter. Le jeu est alors davantage mené pendant les pauses. Il n'introduit pas une hétérogénéité dans l'activité de travail, mais apparaît plutôt dans ce contexte comme une façon de s'occuper lorsqu' on « décroche » ou « sature ».

Dans les deux cas, on observe un effort fait pour désynchroniser des occupations - jeu et travail - qui seraient sinon concurrentes. Cet entrelacement prend une forme différente selon le degré d'homogénéité de l'activité de travail, qui s'avère structurante pour l'activité de jeu. Mais c'est chaque fois en inscrivant jeu et travail dans une même de rythmicité - qu'elle soit celle d'une activité homogène ou hétérogène - qu'ils sont rendus commensurables. Ainsi, le jeu au travail est un temps de jeu, et ce temps a la forme d'une rythmicité. La notion de rythmicité, contrairement à celle d'interactivité, permet de décrire des relations lentes et discontinues, mais produisant une unité de l'expérience. À cette aune, le travail apparaît luimême comme un composite d'activités, plus ou moins enchainnées ou entremêlées, avec des moments de tension et de relâche, enchevêtrement dans lequel la pratique de jeu peut trouver sa place de façon fluide. Aussi, le problème des travailleurs est moins de synchroniser ou d'articuler des temps déjà donnés, comme le temps organisationnel et leur rythme biologique, que de construire une rythmicité personnelle à partir des contraintes sociales pesant sur leurs différentes activités, et les aménageant.

L'étude du jeu au travail donne ainsi à voir les petits arrangements par lesquels les travailleurs tissent leurs préoccupations avec les sollicitations issues du travail. Les activités apparemment les plus personnelles sont bien socialisées - que ce soit dans le couple, la famille, les amis, comme dans le milieu de travail ou d'autres communautés de pratique. Et les temps personnels sont des temps organisés, au sens où ils intègrent les contraintes d'autres intervenants et d'autres contextes. Et si chaque activité engage autant un ensemble de contraintes que des aménagements possibles, «jouer sur le lieu de travail » n'est pas amener le jeu au travail, mais au contraire élaborer sur les lieux une nouvelle manière de s'y prendre. Elle passe notamment par le choix entre «être préoccupé » et «être sollicité »: avoir à y penser ou recevoir une notification ?

Pour chacun, dans un contexte où les espaces-temps du travail sont devenus plus flous (Bidet \& Schoeni, 2011 ; Borzeix \& Cochoy, 2008 ; Crague, 2003 ; Hochschild, 1997), la quête d'une unité de l'expérience repose donc sur la recherche d'une rythmicité personnelle qui compose avec la diversité de ses activités, leurs contraintes et leurs plasticités possibles. L' «indifférence pratique », décrite dans les deux cas précédents, devient dans la forme d'activité de jeu examinée ici la règle et la coordination des travailleurs repose avant tout sur les rythmes qu'ils partagent. 


\section{6.- Conclusion. L'activité au prisme des transactions}

Cet article s'est intéressé à un apport de la notion de transaction introduite par J. Dewey. Il montre qu'une entrée par les transactions permet d'étudier, au-delà du domaine des interactions, un ensemble de situations où l'intelligibilité mutuelle n'est pas centrale pour le déploiement d'une activité collective.

À travers les trois exemples d'une salle de contrôle du réseau téléphonique, d'un service d'urgences pédiatriques et de situations de jeu au travail, nous avons identifié des configurations stabilisées, opérantes, mais prenant en défaut l'horizon de réciprocité des perspectives. Dans les cas étudiés, le déficit d'intelligibilité mutuelle n'est pas préjudiciable à la poursuite de l'activité collective, comme le seraient des «malentendus » (Grosjean, 2005). Nous allons même plus loin en montrant que la coopération y requiert au contraire une faible intelligibilité mutuelle.

Ce modèle enrichit l'étude des contextes organisés, où les environnements de travail à faible degré d'intersubjectivité sont en effet loin d'être anecdotiques. Ils tendent même à se multiplier avec l'approfondissement de la division sociale du travail, qui augmente le nombre de mondes professionnels dont l'activité nous est étrangère, mais avec lesquels nous frayons. La notion de transaction, plus englobante que celle d'interaction, permet de documenter cet aspect du «désenchantement du monde» pointé par M. Weber. Suivant J. Dewey, K. Burke soulignait déjà la difficulté à devenir un «participant» dans un monde « fragmenté » par une «grande diversité professionnelle»: nos apprentissages et savoirs faire pointus risquent toujours de nous «rendre aveugles» (1983, p. 7), et «les différences de façons de vivre et de gagner sa vie », menacent de transformer «les différentes classes d'individus en 'mystères' les uns pour les autres » (p. 276, nous traduisons). Dans un monde fait de tant de «perspectives disparates », devenir un participant ne serait pas tant pour K. Burke une affaire de connaissance que d'action, d'art et d'effort pratique, à travers en particulier le caractère synthétique de nos actes, qui sont chaque fois « une nouvelle manière de mettre des choses ensemble»(1983, p. 254). Documenter cette recherche d'une continuité de l'expérience amène, nous l'avons vu, à étudier la création d'habitudes, de formes de vie, de façons de s'orienter, etc.

Ce modèle élargi au-delà des interactions fait ainsi plus facilement place aux dispositifs numériques, aux tiers et aux rythmes personnels. Les objets, déjà traités comme des appuis inscrits dans l'environnement de travail, apparaissent pour certains animés d'une vie propre, autour de laquelle s'élaborent de véritables formes de vie; les tiers, traditionnellement et presque par définition exclus de l'analyse du travail, se révèlent des opérateurs indispensables à la coopération productive ; enfin, la temporalité de l'activité, en ne cessant de tisser différents rythmes les uns avec les autres, intègre à l'analyse du travail les espacetemps hors travail.

Formes de vie, coïncidence des engagements, entrelacement temporel des activités: les phénomènes mis au premier plan par la notion de transaction amènent à étudier l'activité collective au-delà de l'intelligibilité mutuelle. À partir de situations de travail présentant des similarités avec celles présentées ici, M. Grosjean se demandait si les modèles associés à la théorie de l'activité ne fourniraient pas « un cadre plus satisfaisant [que celui de l'action située] pour repenser la question de la pertinence et de l'intelligibilité mutuelle en fonction des systèmes d'activité [des différents acteurs impliqués dans un travail coopératif] quand ceux-ci ne sont pas convergents » (2005). Un retour à la tradition pragmatiste, dont la théorie de l'activité est elle-même héritière (Garreta, à paraitre), nous semble offrir une voie plus féconde en ce sens. Mettant en évidence des formes d'organisation implicites et pourtant opérantes, sinon structurantes, elle pénètre plus avant la «part d'ombre de l'intelligence collective à l'œuvre dans toute situation de coopération»(Joseph, 2004, p. 23). Car si chacun a toujours besoin de comprendre ce qu'il fait, il n'a pas toujours le loisir, ni le besoin, de bien percevoir ce que font les autres. 
Enfin, faire droit à des contextes où une morale de l'intercompréhension et de la rencontre « en personnes » n'apparaît plus centrale, où la coopération productive s'inscrit moins dans une morale de l'interaction que dans une politique des transactions, rejoint l'exploration pragmatiste de la dimension démocratique du travail (Crick, 2010; Sennett, 2010). L' «indifférence pratique » amène en effet à tolérer la différence, et même l'étrangeté, suivant une configuration politique que l'École de Chicago associait déjà à la grande ville moderne.

\section{BIBLIOGRAPHIE}

Barad, K., (2003). Posthumanist Performativity: toward an understanding of how matter comes to matter. Signs: Journal of Women in Culture and Society, 28.

Barbier, J.-M., \& Durand, M. (Eds.) (2006). Sujets, activités, environnements. Approches transverses. Paris: PUF.

Bechky, B. A. (2003). Sharing Meaning across Occupational Communities: The Transformation of Understanding on a Production Floor. Organization Science, 14(3), 312-330.

Beck U. (2001). La société du risque. Sur la voie d'une autre modernité. Paris, Aubier (1986).

Béguin, P., \& Clot, Y. (2004). L'action située dans le développement de l'activité, @ctivités, 1 (2), 27-49. http://www.activites.org/v1n2/beguin.fr.pdf

Berger, M. (2008), Répondre en citoyen ordinaire. Pour une étude ethnopragmatique des compétences profanes. Tracés, 15, Pragmatismes. Lyon: ENS LSH, 191-208.

Bergman, M. (2007). The new wave of pragmatism in communication studies. Nordicom Review, 29(2), 135-153.

Bidet, A. (2008). L'homme et l'automate. L'écologie élargie du travail contemporain. Sociologie du travail, 50(3), 372-395.

Bidet, A. (2010). Qu'est-ce que le vrai boulot? Le cas d'un groupe de techniciens. Sociétés contemporaines, 78, 115-135.

Bidet, A. (2011a). L'engagement dans le travail. Qu'est-ce que le vrai boulot ? Paris: PUF.

Bidet, A. (2011b). La multi-activité, ou le travail est-il encore une expérience ? Communications, 89. Paris: Éditions de l'EHESS, 9-26.

Bidet, A. (2012). Activité. In A. Bevort, A. Jobert, M. Lallement, \& A. Mias (Eds.), Dictionnaire $d u$ travail (pp. 6-12). Paris: PUF, Quadrige.

Bidet, A., \& Schoeni, D. (2011). Décrire les présences au travail, analyser la structuration de la vie sociale. Ethnographiques.org, 23 (http://www.ethnographiques.org/2011/ Bidet,Schoeni — consulté le 19.01.2013)

Borzeix, A., \& Cochoy, F. (2008). Travail et théorie de l'activité : vers des «workspace studies »? Sociologie du travail, 51(3), 273-286.

Boutet, M. (2006). L'ordinateur à l'état sauvage. Une approche écologique. In A. Bidet, A. Borzeix, T. Pillon, G. Rot, \& F. Vatin (Eds.), Sociologie du travail et activité (pp. 29-45). Toulouse: Octarès, Coll. Le travail en débats.

Boutet, M. (2008). S'orienter dans les espaces sociaux en ligne. L'exemple d'un jeu. Sociologie du travail, 50(4), 447-470.

Boutet, M. (2011). Un rendez-vous parmi d'autres. Ce que le jeu sur internet nous apprend du travail contemporain. Ethnographique.org, 23, (http://www.ethnographiques.org/2011/ Boutet consulté le 19.01.2013)

Breviglieri, M. (1997). La coopération spontanée. Entraides techniques autour d'un automate public. In B. Conein, \& L. Thevenot (Eds.), Cognition et information en société (pp.). Paris: EHESS, Raisons pratiques 8.

Breviglieri M. (2004). Habiter l'espace de travail. Perspectives sur la routine. Histoire \& Socités, 9 , 19-29.

Breviglieri, M. (2010). L' «épuisement capacitaire » du sans-abri comme urgence ? Approche phénoménologique du soin engagé dans l'aide sociale (gestes, rythmes et tonalités d'humeur). In C. Felix, \& J. Tardif (Eds.), Actes éducatifs et de soins, entre éthique et gouvernance, Nice 4-5 
juin 2009, URL : http://revel.unice.fr/symposia/actedusoin/index.html?id=795.

Breviglieri, M., \& Stavo-Debauge, J. (2007). L'hypertrophie de l'oeil. Pour une anthropologie du «passant singulier qui s'aventure à découvert ». In D. Cefaï, \& C. Saturno (Eds.), Itinéraires d'un pragmatiste. Autour d'Isaac Joseph (pp. 79-98). Paris: Economica.

Burke, K. (1983). Permanence and change. An Anatomy of Purpose. Berkeley: University of California Press (1935).

Cefaï, D. (1998). Phénoménologie et sciences sociales: Alfred Schutz, naissance d'une anthropologie philosophique. Paris et Genève: Librairie Droz.

Chateauraynaud, F. (1997). Vigilance et transformation. Présence corporelle et responsabilité dans la conduite des dispositifs techniques. Réseaux, 85, 101-127.

Chave, F. (2010). Tiers en urgences. Les interactions de secours, de l'appel au 18 à l'accueil en service d'urgences pédiatriques. Contribution à une sociologie du tiers. Thèse de doctorat, sous la direction d'A. Borzeix. Nanterre: Université Paris Ouest-Nanterre La défense, 13 décembre 2010.

Cicourel, A. (1987). Le raisonnement médical. Une approche sociocognitive. Paris: Seuil.

Crague, G. (2003). Des lieux de travail de plus en plus variables et temporaires. Économie et statistique, 369-370, 191-212.

Craig, R. T. (2007). Pragmatism in the field of communication theory. Communication Theory, 17, $125-145$.

Crick, N. (2010). Democracy \& Rhetoric. John Dewey and the Arts of Becoming. Columbia: The University of South Carolina Press. .

Datchary, C., \& Licoppe, C. (2007). La multi-activité et ses appuis. L'exemple de la «présence obstinée » des messages dans l'environnement de travail. @ ctivités, 4(1), 4-29.

Descombes, V., (1995), La denrée mentale. Paris: Minuit.

Denis, J., \& Pontille, D. (2010). Petite sociologie de la signalétique. Les coulisses des panneaux du métro. Paris: Presses des Mines.

Dewey, J. (1993). Logique. Théorie de l'enquête. Paris: PUF (1938).

Dewey, J., (2005). L'art comme expérience. Pau: Publications de l'Université de Pau-Farrago (1934).

Dewey, J. (2011a). Démocratie et éducation. Suivi de Expérience et éduction. Paris: Armand Colin (1916).

Dewey, J. (2011b). La formation des valeurs (traduction et présentation par A. Bidet, L. Quéré, \& G. Truc). Paris: La Découverte.

Dodier, N. (1995). Les hommes et les machines. La conscience collective dans les sociétés technicisées. Paris: Métailié.

Dourish, P. (2001). Where the Action Is: The Foundations of Embodied Interaction. Cambridge: MIT Press.

Dreyfus, H. (1991). Being-in-the-world. Cambridge: MIT Press.

Engeström, Y. (2008). Quand le centre se dérobe : la notion de knotworking et ses promesses. Sociologie du travail, 50(3), 303-330.

Emirbayer, M. (1997). Manifesto for a Relational Sociology. The American Journal of Sociology, 103(2), 281-317.

Fligstein, N., \& McAdam, D. (2012). A Theory of Fields. Oxford: Oxford University Press.

Garreta, G. (à paraitre). Pragmatisme et pédologie. Dewey, Vygotski et la pédagogie soviétique des années 1920. In J. Friedrich, R. Hofstetter, \& B. Schneuwly (Eds.), Une science du développement est-elle possible? Aspects historiques.

Goffman, E. (1979). Asiles. Études sur la condition sociale des malades mentaux et autres reclus. Trad. de Liliane et Claude Lainé, Paris: Éditions de Minuit.s[

Goodwin, C., \& Goodwin, M.H. (1996). Formulating Planes. Seeing as a situated activity. In Y. Engeström, \& D. Middleton (Eds.), Cognition and communication at work (pp. 61-95). Cambridge: Cambridge University Press.

Grafmeyer, Y., \& Josepth, I. (2004). L'école de Chicago. Naissance de l'écologie urbaine. Paris: Flammarion Champ. 
Grosjean, M. (2005). L'awareness à l'épreuve des activités dans les centres de coordination. @ctivités, 2(1), 76-98, http://www.activites.org/v2n1/grosjean.pdf

Grosjean, M., \& Lacoste, M. (1999). Communication et intelligence collective. Le travail à l'hôpital. Paris: Presses Universitaires de France, coll. Le Travail humain.

Gumperz, J. (1989). Engager la conversation. Introduction à la linguistique interactionnelle. Paris: Minuit.:SEP:DE:

Heath, C., Hindmarsh, J., \& Luff P. (Eds.) (2000). Workplace studies. New York and Cambridge: Cambridge University Press.

Hennion, A. (2009). Réflexivités. L'activité de l'amateur. Réseaux, 153, 55-78.

Hochschild, A. R. (1997). The Time Bind: When Work Becomes Home and Home Becomes Work. New York: Metropolitan Books.

Hughes, E.C. (1996). Le regard sociologique. Essais choisis. Textes rassemblés et présentés par J.-M. Chapoulie. Paris: Editions EHESS.

Ingold, T. (2001). The Perception of Environment: Essays in Livelihood, Dwelling and Skill. London: Routledge.

Joas, H. (1999). La créativité de l'agir. Paris: Cerf (1992).

Joseph, I. (1994). Attention distribuée et attention focalisée. Les protocoles de la coopération au PCC de la ligne A du RER, Sociologie du travail, 36(4), 563-585.

Joseph, I. (2002). Pluralisme et contiguités. In I. Joseph, \& D. Cefaï (Eds.), L’héritage du pragmatisme. Conflits d'urbaniste et épreuves du civisme (pp. 83-105). La Tour d'Aigues: Editions de l'Aube.

Joseph, I. (2004). L'athlète moral et l'enquêteur modeste. pp. 19-52. In B. Karsenti, \& L. Quéré (Eds.), La croyance et l'enquête. Aux sources du pragmatisme (pp.). Paris: EHESS, Raisons pratiques.

Joseph, I. (2007a). Simmel, l'écologie urbaine et Goffman. In D. Cefaï, \& C. Saturno (Eds .), Itinéraires d'un pragmatiste. Autour d'Isaac Joseph (pp. 3-18). Paris: Economica.

Joseph, I. (2007b). L'athlète moral et l'enquêteur modeste (restes réunis par D. Cefaï). Paris: Economica.

Joseph, I., \& Quéré, L. (1993). L'organisation sociale de l'expérience. Futur antérieur, 19-20, 137 150.

Kaptelinin, V., \& Nardi, B.A. (2006). Acting with technogy. Activity theory and interaction design. Cambridge: MIT Press.

Katz, J. (1999). How Emotions work. Chicago: The University of Chicago Press.

Katz, J. (2002). Start Here: Social Ontology and Research Strategy. Theoretical Criminology, 6(3), 255-278.

Kendon, A. (1990). Conducting Interaction: Patterns of Behavior in Focused Encounters. Cambridge: Cambridge University Press.

Kilpinen, E. (2009). The Habitual Conception of Action and Social Theory. Semiotica, 173 (1/4), 99128.

Knorr-Cetina, K. (1997). Sociality with Objects : Social relations in Postsocial Knowledge Societies. Theory, Culture \& Society, 14(4), 1-30.

Knorr-Cetina, K. (1998). Les épistémès de la société : l'enclavement du savoir dans les structures sociales. Sociologie et Sociétés, 30(1), 39-54.

Knorr-Cetina, K., \& Bruegger, U. (2003). La technologie habitée. La forme de vie globale des marchés financiers. Réseaux, 21(122), 111-132.

Lahlou, S. (2000). La cognition au travail et ses outils : débordement, révolution, distribution, Les attracteurs cognitifs et le syndrome du débordement. Intellectica, 30(1), 75-113.

Licoppe, C. (2008). Logiques d'innovation, multiactivité et zapping au travail. Hermès, 50, 171-178.

McKenzie, R.D. (1924). The eco logical approach to the study of huamna community. In R.E. Park, E.W. Burgess, \& T.D. McKenzie (Eds), The City (pp. 63-79).Chicago: University of Chicago Press.

Nardi, B., \& O’Day, V. (1999). Information ecologies : Using Technologies with Heart. Cambridge: 
MIT Press.

Norros, L. (2004). Acting under Uncertainty. The Core-Task Analysis in Ecological Study of Work. Espoo: VTT.

Pagis, M. (2010). Producing intersubjectivity in silence : an ethnographic study of meditation practice. Ethnography, 11 (2), 309-328.

Peneff, J. (2000). Les malades des urgences. Paris: Métaillié.

Piette, A. (2009a). Anthropologie existentiale. Paris: Éditions Pétra, coll. « Anthropologiques ».

Piette, A. (2009b). L'acte d'exister. Une phénoménographie de la présence. Paris: Socrate Editions.

Quéré, L. (1997). La situation toujours négligée ? Réseaux (85).

Quéré, L. (2006). L'environnement comme partenaire. In J. M. Barbier, \& M. Durand (Eds.), Sujets, activités, environnements. Approches transverses (pp. ). Paris: PUF.

Quéré, L., \& Terzi, C. (2011). Some Features of Pragmatist Thought Still Remain Insufficiently Explored in Ethnomethodology. Qualitative Sociology, 34 (1), 271-275.

Rosa, H. (2010). Accélération. Une critique sociale du temps. Traduction française. Paris: La Découverte (2005).

Russill, C. (2008). Through a Public Darkly : Reconstructing Pragmatist Perspectives in Communication Theory. Communication Theory, 18, 478-504.

Salembier, P., \& Zouinar, M. (2004). Intelligibilité mutuelle et contexte partagé, Inspirations conceptuelles et réductions technologiques, @ctivités, 1(2), 64-85. http://www.activites.org/v1n2/salembier.pdf

Schatzki T.R., Knorr Cetina K., von Savigny E. (eds.) (2001). The Practice Turn in Contemporary Theory. London: Routledge.

Schmidt, K. (2012). The trouble with 'Tacit Knowledge'. Computer Supported Cooperative Work, 21, 163-225.

Sennett, R. (2010), Ce que sait la main. La culture de l'artisanat. Traduction française, Paris : Albin Michel (2008).

Simondon, G. (1989). L'individuation psychique et collective. Paris: Aubier (1958).

Simpson, B. (2009), Pragmatism, Mead and the Pratice Turn. Organization Studies, 30(12), 13291347 (traduction dans ce volume).

Strauss, A. (1992). La Trame de la négociation. Paris: L'Harmattan.

Strauss, A. (1993). Continual Permutations of Action, New Brunswick \& London: Transaction Publishers.

Suchman, L. (2007). Human-Machine Reconfigurations. Cambridge: Cambridge University Press.

Tracés. Revue de Sciences Humaines (2008). Pragmatismes, 15. Lyon: ENS LSH.

Tracés. Revue de Sciences humaines (2012). Écologiques. Enquêtes sur les milieux humains, 22. Lyon: ENS LSH.

\section{RESUME}

La notion d'interaction, au cœur de bien des approches issues de la tradition pragmatiste, a nourri des recherches focalisées sur la production d'intelligibilité mutuelle entre les participants, notamment dans le champ du travail. Cet article montre qu'il convient toutefois d'introduire une notion plus large, celle de transaction, pour caractériser l'apport de cette tradition. On peut alors saisir son actualité pour l'étude du travail dans nos sociétés de plus en plus technicisées et cosmopolites, en particulier là où l'activité est menée par des participants aux perspectives radicalement hétérogènes. Trois études de cas, respectivement dans un centre de contrôle du trafic téléphonique, dans des services pédiatriques de soins d'urgence, et sur la pratique de jeux en ligne au travail, viennent illustrer notre argument. Ces activités 
collectives ne reposent pas sur la recherche d'une intelligibilité mutuelle, mais sur des formes de vie développées au travail, des rencontres ponctuelles entre des engagements qui s'ignorent et l'entrelacement par chacune des activités. Dans ces contextes, la notion de transaction permet de porter une attention particulière au rôle des dispositifs numériques, des tiers et des rythmes personnels.

\section{MOTS-CLES}

coopération, activité collective, travail, expérience, transaction, pragmatisme, sociologie, urgence, hôpital, jeu, supervision de trafic

\section{REFERENCEMENT}

Bidet, A., Boutet, M., \& Chave, F. (2013). Au-delà de l'intelligibilité mutuelle : l'activité collective comme transaction. Un apport du pragmatisme illustré par trois cas. Activités, 10(1), 172-191, http://www.activites.org/v10n1/v10n1.pdf

Article soumis le 8 septembre 2012, accepté pour publication le 6 décembre 2012. 